\title{
20(S)-Protopanaxadiol saponins inhibit SKOV3 cell migration
}

\author{
BIN LI ${ }^{1}$, DAOMEI CHEN ${ }^{1}$, WANYI LI ${ }^{2}$ and DAN XIAO ${ }^{2}$ \\ ${ }^{1}$ Key Laboratory of Medicinal Chemistry for Natural Resources, Ministry of Education, School of Pharmacy, \\ School of Chemical Sciences and Technology, Yunnan University, Kunming, Yunnan 650091; \\ ${ }^{2}$ Institute of Medicinal Plants, Yunnan Academy of Agricultural Sciences, Kunming, Yunnan 650223, P.R. China
}

Received September 28, 2014; Accepted January 5, 2016

DOI: $10.3892 / 01.2016 .4155$

\begin{abstract}
While the anti-tumor actions of ginsenosides from Panax notoginseng are well-studied, the anti-proliferative activity of 20(S)-protopanaxadiol saponins (PDS) in Sanchi ginseng on human ovarian cancer has not been reported, nor has its effect on migration of SKOV3 cells been investigated. In the present study, a wound-healing assay indicated that PDS inhibited the migration of SKOV3 cells, and a Matrigel ${ }^{\mathrm{TM}}$ tube formation assay demonstrated the presence of inhibitory tube-structures following treatment with PDS. To date, there are no previous reports on the regulation of osteopontin (OPN), a glycophosphoprotein cytokine frequently expressed in ovarian carcinoma effusions by PDS. A reduction in the expression of OPN following PDS-treatment was observed using immunohistochemical and western blot experiments. These results suggest that PDS may be useful in the search for a potential ovarian cancer treatment.
\end{abstract}

\section{Introduction}

Ovarian cancer is one of the most prevalent gynecological neoplasms and major cause of cancer-associated mortality in women. The five-year survival rate remains $<40 \%$ for stage IIB to IV ovarian cancers, despite advancements in surgical techniques and treatments (1). Thus, novel and more effective agents for the treatment of ovarian cancer are required.

Panax notoginseng, a common Chinese herb, is known to contain ginsenosides, a class of compounds with a number of biological activities, including antioxidation (2), immunomodulatory effects (3) and anticancer properties $(4,5)$. The ability of ginsenosides to inhibit metastasis has been demonstrated by strong pleiotropic anti-cancer effects observed previously in several cancer lines $(6,7)$. The aglycone compounds

Correspondence to: Mrs. Bin Li, Key Laboratory of Medicinal Chemistry for Natural Resources, Ministry of Education, School of Pharmacy, School of Chemical Sciences and Technology, Yunnan University, 2 Chuihu Road, Kunming, Yunnan 650091, P.R. China E-mail: libin36@ynu.edu.cn

Key words: Panax notoginseng saponins, migration, ostopontin, ovarian cancer, SKOV3 cells, tube formation of ginsenosides have been seldom studied, despite intensive research into the physiological activities of ginsenosides.

Osteopontin (OPN) is a protein that is overexpressed in ovarian cancer, its expression is frequently identified in ovarian carcinoma effusions, and it has an involvement in tumorigenesis and metastasis $(8,9)$. OPN silencing by small interfering RNA suppresses the proliferation of human ovarian cancer cell line HO-8910PM in vitro (10), suggesting that increased OPN expression may be key in the carcinogenesis, progression, and differentiation of epithelial ovarian carcinoma. Regulation of OPN activity may therefore be useful in ovarian cancer therapy. However, to date there are no reports on the regulation of OPN expression using ginseng extracts, such as 20(S)-protopanaxadiol saponins (PDS).

The aim of the present study was to investigate the anti-tumour effects of PDS and conduct a preliminary study of its mechanisms of action. This included studying the effects of PDS on the migration of SKOV3 cells, and the effects of PDS on the expression of OPN.

\section{Materials and methods}

Materials and chemicals. 20(S)-protopanaxadiol saponins from Panax notoginseng (Burkill) F. H. Chen, with ginsenosides Rb1, Rb3 and $\mathrm{Rd}$ (56.42, 22.99 and 13.40\%, respectively) were obtained from Kunming Pharmaceutical Corp. (Kunming, China). An immunohistochemistry analysis kit was purchased from Zhongshan Golden Bridge Biotechnology Co., Ltd (Beijing, China). Gibco ${ }^{\circledR}$ RPMI-1640 medium was purchased from Thermo Fisher Scientific, Inc. (Waltham, MA, USA). Fetal bovine serum (FBS), penicillin and streptomycin were purchased from Sigma-Aldrich (St. Louis, MO, USA) and Matrigel ${ }^{\mathrm{TM}}$ was obtained from BD Biosciences (Franklin Lakes, NJ, USA). Crystal violet and polylysine were purchased from Beyotime Institute of Biotechnology (Beijing, China). SP9001 rabbit streptavidin peroxidase (SP) test kit was purchased from Zhongshan Jinqiao Biotech Company (Beijing, China). The following antibodies were used: Mouse anti-human monoclonal pro-caspase-3 (catalog no., sc-56053; Santa Cruz Biotechnology, Inc., Dallas, TX, USA), mouse anti-human monoclonal B-cell lymphoma (Bcl)-2 (catalog no., sc-7382; Santa Cruz Biotechnology, Inc.), mouse anti-human monoclonal Bcl-2-like protein 4 (Bax) (catalog no., sc-70405; Santa Cruz Biotechnology, Inc.), rabbit anti-human polyclonal OPN (catalog no., SAB2700986; Sigma-Aldrich, St. Louis, 
MO, USA), mouse monoclonal anti-human $\beta$-actin (catalog no., sc-47778; Santa Cruz Biotechnology Inc., Dallas, TX, USA) and HRP-labeled goat anti-rabbit and anti-mouse immunoglobulin (Ig)G (catalog nos., A0208 and A0216, respectively; dilution, 1:1,000; Beyotime Institute of Biotechnology). All chemicals used were of reagent or analytical grade.

Cancer cell lines and cell culture. The SKOV3 ovarian cancer cell line, obtained from the American Type Culture Collection (Manassas, VA, USA) was cultured in RPMI-1640 containing $10 \% \mathrm{FBS}$ and $1 \%$ penicillin/streptomycin in a humidified atmosphere with $5 \% \mathrm{CO}_{2}$ at $37^{\circ} \mathrm{C}$.

Determination of cytotoxicity of SKOV3 cells. The in vitro anti-proliferative effect of PDS on SKOV3 cells was examined using 3-(4,5-dimethylthiazol-2-yl)-2,5-diphenyltetrazolium bromide (MTT; Thermo Fisher Scientific, Inc.) assay. Cells were placed in 96 -well plates $\left(6 \times 10^{3}\right.$ cells/well $)$ and subsequently were treated with $0.013,0.025,0.05,0.1,0.2$ and $0.4 \mathrm{mg} / \mathrm{ml}$ of PDS. Following a treatment time of 12, 24 or $48 \mathrm{~h}, 25 \mu \mathrm{l}$ MTT solution $(5 \mathrm{mg} / \mathrm{ml})$ was added to each well and incubated at $37^{\circ} \mathrm{C}$ in $5 \% \mathrm{CO}_{2}$ for $4 \mathrm{~h}$. Dimethyl sulfoxide (150 $\mu \mathrm{l}$; Thermo Fisher Scientific, Inc.) was added to dissolve any crystal formation. The cytotoxicity against cancer cells was determined by measuring the absorbance of the MTT at $490 \mathrm{~nm}$ using a Spectra Max 190 microplate spectrophotometer (Molecular Devices Corporation, Sunnyvale, CA, USA). Cytotoxicity of each sample was expressed as an $\mathrm{IC}_{50}$ value. The $\mathrm{IC}_{50}$ value is the concentration of test sample that causes $50 \%$ inhibition of cell growth, averaged from 3-5 replicate experiments, and was obtained by plotting the percentage inhibition vs. concentration of a test sample. Oxaliplatin (OXA; Jiangsu Aosaikang Pharmaceutical Co., Ltd., Nanjing, China) was used as a positive control, which is an effective anticancer drug. The results are expressed as the mean \pm standard deviation (SD) of three independent experiments.

Wound-healing migration assay. A confluent single-layer of cells was seeded onto polylysine-coated $(100 \mu \mathrm{g} / \mathrm{ml})$ coverslips and the cells were left to adhere overnight in the incubator. Scraping a sterile P200 pipette tip across the cells produced a wound in the monolayer. Subsequently, the cells were treated with $0.2 \mathrm{mg} / \mathrm{ml}$ PDS for $0,6,12$ or $24 \mathrm{~h}$. Cell debris was removed by washing the coverslips once with sterile phosphate-buffered saline (PBS), and cells were fixed by incubation with $80 \%$ ethanol for $10 \mathrm{~min}$ at room temperature. The cells were stained with crystal violet and migrating cells were counted in 5 regions. Images were captured using an IX71 Inverted Microscope with a DP73 digital camera (Olympus Corp., Tokyo, Japan; magnification, x40). Cell migration was expressed as the mean $\pm \mathrm{SD}$ of 5 independent experiments.

Tube structure formation assay. An in vitro Matrige ${ }^{\mathrm{TM}}$ substratum assay was used to measure tube structure formation. A 96-well plate (Thermo Fisher Scientific, Inc.) coated with a total of $50 \mu \mathrm{L}$ of Matrigel ${ }^{\mathrm{TM}}$ basement membrane matrix was incubated for $1 \mathrm{~h}$ at $37^{\circ} \mathrm{C}$. A total of $2 \times 10^{4}$ SKOV3 cells in $100 \mu \mathrm{l}$ of medium were seeded into the wells, and $0.2 \mathrm{mg} / \mathrm{ml}$ or $0.4 \mathrm{mg} / \mathrm{ml}$ PDS was then added. Tube-like SKOV3 cell structures formed after $6 \mathrm{~h}$ of incubation at $37^{\circ} \mathrm{C}$ in $5 \% \mathrm{CO}_{2}$ were recorded using an IX71 Inverted Microscope (magnification, x40).

Immunohistochemical analysis of OPN expression. Immunohistochemistry analysis was performed using the SP9001 rabbit SP test kit, according to manufacturer's protocol. The immunoreaction was visualized using 3, 3'-diaminobenzidine (DAB; Zhongshan Jinqiao Biotech Company) staining (yellow or brown visualization). The cells were counterstained with hematoxylin (blue visualization; Beyotime Institute of Biotechnology). Briefly, cells were plated onto coverslips and treated with $0.2 \mathrm{mg} / \mathrm{ml}$ PDS in serum-free RPMI-1640 for $24 \mathrm{~h}$ and subsequently fixed with $80 \%$ ethanol for $10 \mathrm{~min}$. The cells were incubated with polyclonal anti-OPN antibodies (dilution, 1:400) for $1 \mathrm{~h}$ at room temperature, followed by incubation with HRP-labeled goat anti-rabbit IgG for $15 \mathrm{~min}$, washed with PBS three times, and subsequently incubated with streptavidin-horseradish peroxidase (Zhongshan Jinqiao Biotech Company) for $15 \mathrm{~min}$. The cells were washed with PBS three times, were stained with DAB for 8 min and washed with distilled water for $2 \mathrm{~min}$. The cells were stained with hematoxylin and washed with PBS three times, then examined and photographed with an IX71 Inverted Microscope with a DP73 digital camera.

Western blot analysis. The expression levels of four proteins, Bcl-2, Bax, pro-caspase-3 and OPN, were evaluated using western blot analysis. SKOV3 cells $\left(1 \times 10^{5}\right.$ cells/well) were seeded in 6-well plates (Thermo Fisher Scientific, Inc.) and were treated with various concentrations of PDS for 6 or $12 \mathrm{~h}$. The medium was removed and the cells were washed with PBS. The cells were lysed in $100 \mu 1$ lysis buffer (Beyotime Institute of Biotechnology) for $30 \mathrm{~min}$ on ice. The lysates were centrifuged (Eppendor $\mathrm{f}^{\circledR}$ Multipurpose centrifuge 580R; Eppendorf, Hambur, Germany) at $15,000 \mathrm{x} g$ for $15 \mathrm{~min}$. A protein quantification assay kit was obtained from Bio-Rad Laboratories (catalog no., 5000001; Bio-Rad Laboratories, Hercules, CA, USA). Absorbance was determined using a Spectra Max 190 microplate spectrophotometer. Proteins $(30 \mu \mathrm{g})$ were resolved using electrophoresis on a 4-12\% sodium dodecyl sulfate-polyacrylamide gels and were transferred to polyvinylidene fluoride membranes (EMD Millipore,Billerica, MA, USA). Transferred blots were incubated with blocking agents [5\% non-fat milk in PBS-Tween 20 (Thermo Fisher Scientific, Inc.)]. The membranes were incubated with antibodies against pro-caspase-3 (dilution, 1:200), Bcl-2 (dilution, 1:200), Bax (dilution, 1:200), $\beta$-actin (dilution, 1:500) and OPN (dilution, 1:500) overnight. The membranes were subsequently washed 3 times with PBS and incubated with goat anti-rabbit or anti-mouse $\mathrm{IgG}$. The proteins were then detected using Pierce ${ }^{\mathrm{TM}}$ enhanced chemiluminescence Western Blotting Substrate (Thermo Fisher Scientific, Inc.). $\beta$-actin was used as a protein-loading control.

Statistical analysis. In total, 3-5 independent replicates were conducted for all experiments. The results are expressed as the mean $\pm \mathrm{SD}$. The Student's $t$ test with a significance level of $\mathrm{P}<0.05$ was used to calculate statistically significant differences. OriginLab version 8.5 software (OriginLab, Northampton, MA, USA) was used to analyze the experimental data. 

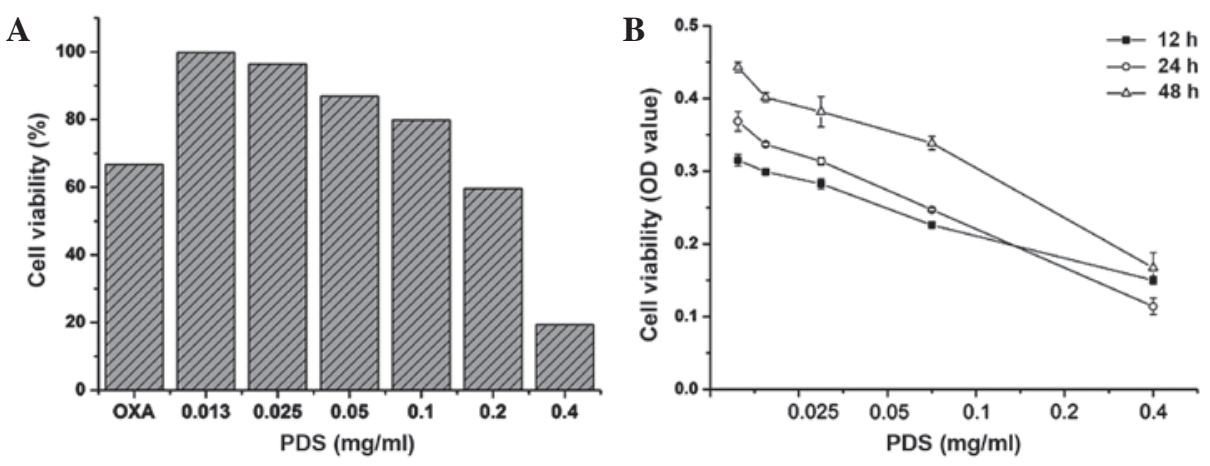

Figure 1. Inhibition of cell viability by PDS in SKOV3 cells. (A) Cells were seeded at an initial density of $6 \times 10^{3}$ cells/well in a 96 -well plate and incubated for $24 \mathrm{~h}$ and treated with various concentrations of PDS for $12-48 \mathrm{~h}$. OXA $(0.2 \mathrm{mg} / \mathrm{ml})$ was used as a positive control. (B) Cell viability as measured by absorbance under various PDS treatments with various incubation times. PDS, 20(S)-protopanaxadiol saponins; OXA, oxaliplatin; OD, optical density.
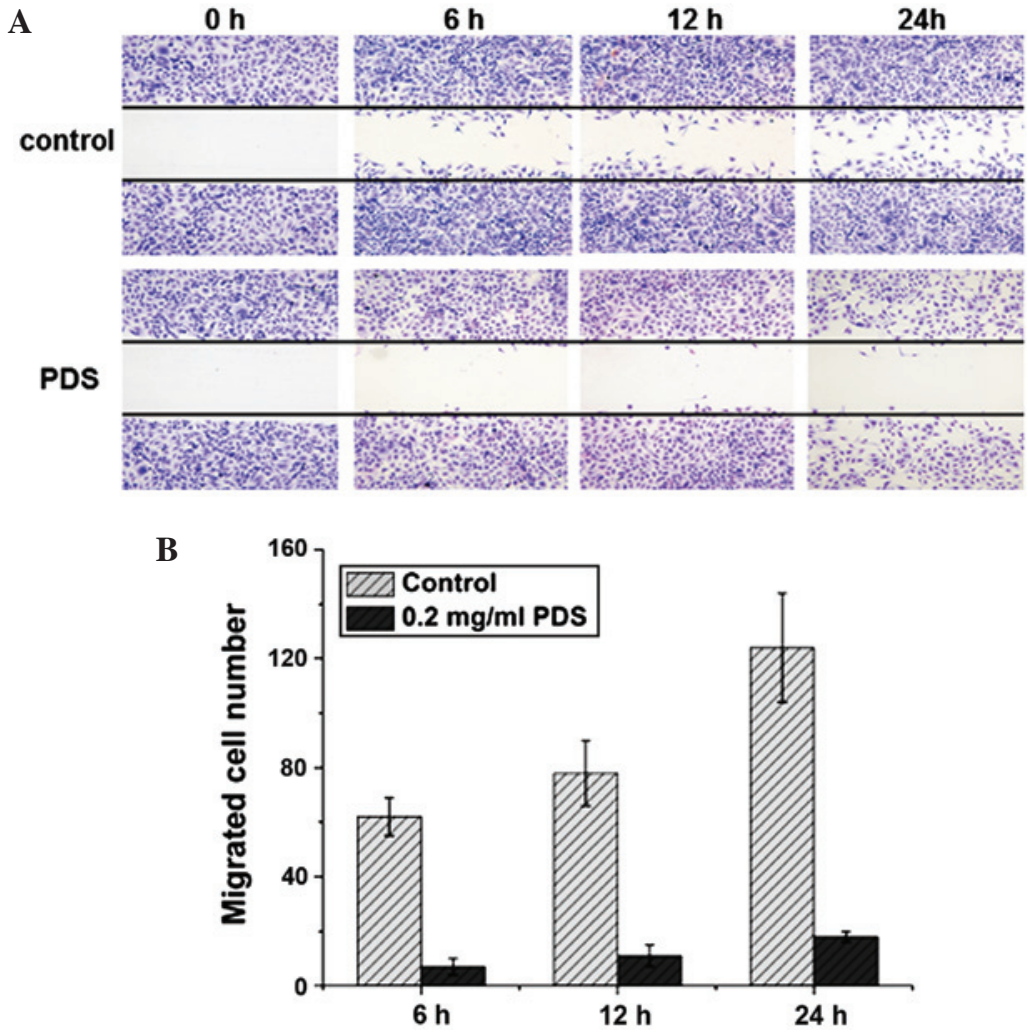

Figure 2. (A) PDS inhibits SKOV3 cells migration in the wound-healing assay. Cells were plated on 60-mm coverslips and grown to a confluent monolayer. A scratch was created by scraping the monolayer with a P200 pipette tip. Cells were stained with crystal violet. Magnification, x40. (B) Quantitative results of migrated cancer cells following PDS treatment $(n=3 ; P<0.05)$. PDS, 20(S)-protopanaxadiol saponins.

\section{Results}

PDS inhibits the viability of SKOV3 cells. The MTT assay demonstrated that PDS significantly inhibited SKOV3 cell proliferation. As shown in Fig. 1, PDS significantly inhibited the viability of SKOV3 cells when treated with various concentrations of SDS $(0-0.4 \mathrm{mg} / \mathrm{ml})$ during various incubation times (12-48 h). Notably, the optimum treatment time was achieved at $24 \mathrm{~h}\left(\mathrm{IC}_{50}=0.30 \mathrm{mg} / \mathrm{ml}\right)$. OXA was used as a positive control. The inhibition rate of the cells by PDS was increased compared with OXA during the early stage of drug treatment, including at a $24 \mathrm{~h}$ treatment time at $0.2 \mathrm{mg} / \mathrm{ml}$ PDS decreased SKOV3 cell viability by $\sim 40 \%$, whereas SKOV3 cell viability decreased by $\sim 27 \%$ following treatment with $0.2 \mathrm{mg} / \mathrm{ml}$ OXA. In addition, cell viability decreased by $\sim 81 \%$ when the cells were treated with $0.4 \mathrm{mg} / \mathrm{ml}$ of PDS, suggesting that PDS exhibits toxic effects at high concentrations. However, following $24 \mathrm{~h}$ treatment, the cytotoxicity of PDS decreased as the treatment time increased. This suggests that PDS has a short-duration cytotoxicity on SKOV3 cells.

The effect of PDS on SKOV3 cell migration. Following treatment with either 0.2 or $0.4 \mathrm{mg} / \mathrm{ml}$ of PDS, wound-healing experiments were conducted and the number of SKOV3 cells migrating to the wound area every $6 \mathrm{~h}$ for 1 day was quantified to determine if PDS inhibits cell migration (Fig. 2A). The 
A
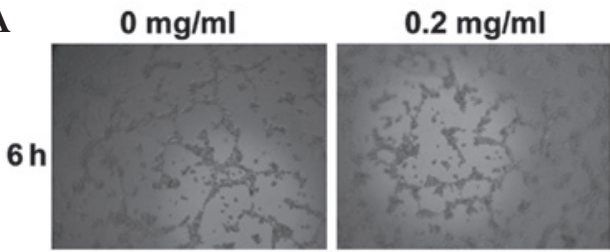

$0.4 \mathrm{mg} / \mathrm{ml}$
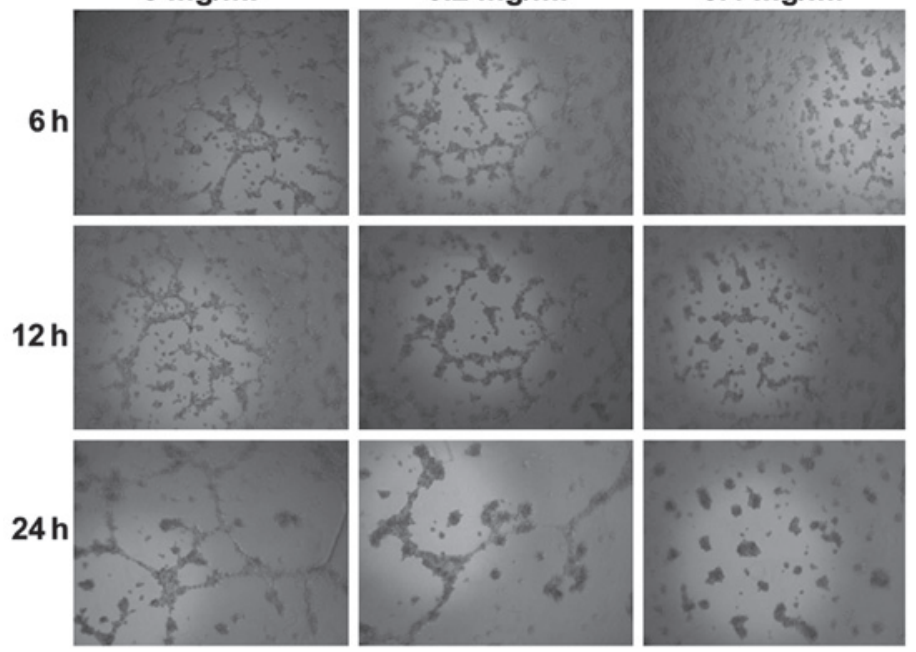

B

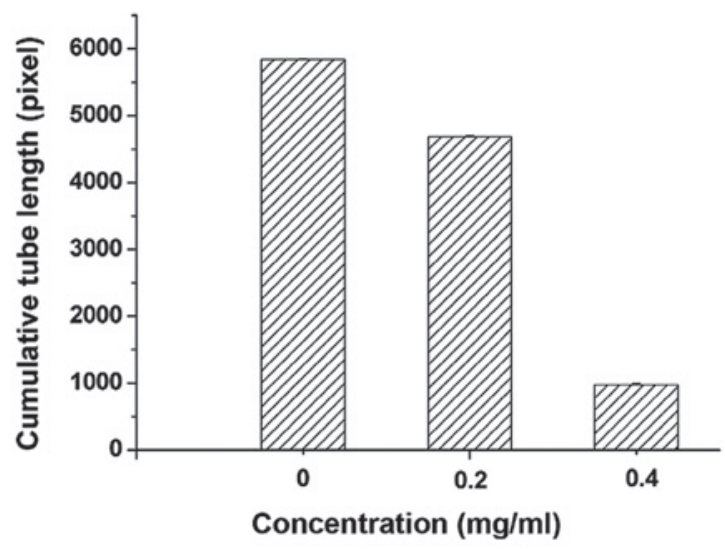

Figure 3. PDS inhibits tube structure formation of SKOV3 cells in Matrigel. PDS exposure inhibited SKOV3 cells differentiation into tubes in vitro in a dose-dependent fashion. (A) Representative fields at PDS concentrations of 0, 0.2 and $0.4 \mathrm{mg} / \mathrm{ml}$. Magnification, $\mathrm{x} 40$. (B) Cumulative tube length in four wells (magnification, $\mathrm{x} 40$ ) for replicate experiments $(\mathrm{P}<0.005)$. PDS, 20(S)-protopanaxadiol saponins.

migrating cells were counted in 5 regions and the results were calculated as the means from 5 replicates of each experiment. As shown in Fig. 2B, PDS inhibited SKOV3 migration in a time-dependent manner. Quantitatively, SKOV3 cell viability was decreased by $\sim 40 \%$ following $24 \mathrm{~h}$ treatment by $0.2 \mathrm{mg} / \mathrm{ml}$ of PDS (Fig. 1B), suggesting the effect of inhibitory SKOV3 migration was not due to cell death.

Tube structure formation is inhibited by PDS in SKOV3 cells. Following incubation of SKOV3 cells in Matrigel ${ }^{\mathrm{TM}}$-coated 96-well plates for $6 \mathrm{~h}$ with medium, the results demonstrate that untreated SKOV3 cells formed well-organized tube-like structures, while PDS incubated cells formed significantly fewer and shorter tubes (Fig. 3A). When the concentration of PDS was increased from 0.2 to $0.4 \mathrm{mg} / \mathrm{ml}$, PDS incubation significantly reduced the average summated length of capillary tubes $(\mathrm{P}<0.005)$. Therefore, SKOV3 cell tube structure formation was inhibited by PDS (Fig. 3B).

Effects of PDS on protein localization of OPN. Immunohistochemical analysis was used to evaluate the expression of OPN in SKOV3 cells. Compared with control cells, PDS-treated cells exhibited a number of morphological changes (Fig. 4A). In particular, the cells shrunk, lost cytoplasm volume and had 'naked nuclei' following $24 \mathrm{~h}$ of $0.4 \mathrm{mg} / \mathrm{ml}$ PDS treatment. OPN was weakly-expressed in PDS-treated cell groups, and the expression was PDS dose-dependent, indicating that the expression of OPN protein was inhibited by PDS (Fig. 4B). The expression of OPN protein was mostly in the cytoplasm of control cells with yellow or brown yellow staining.

Regulation of the expression of several proteins. The changes in protein expression levels following PDS treatment was assessed. The levels of OPN protein were visualized using western blot analysis with protein extracts from PDS-treated cells. The results demonstrate that PDS treatment led SKOV3 cells to downregulate OPN protein expression (Fig. 5). The migration of SKOV3 ovarian cancer cells was significantly inhibited by PDS, and this may be associated with the observed reduction in OPN expression. Furthermore, the protein expression levels of pro-caspase-3, Bcl-2 and Bax were investigated, which are important in regulating and initiating apoptosis in ovarian cancer $(11,12)$. As shown in Fig. 5, the pro-caspase-3 and Bcl-2 protein expression levels were downregulated by PDS and Bax was upregulated. This has also been reported by Park et al (5). Therefore, PDS is a potent antitumor molecule that may be developed as a cancer therapeutic agent. 

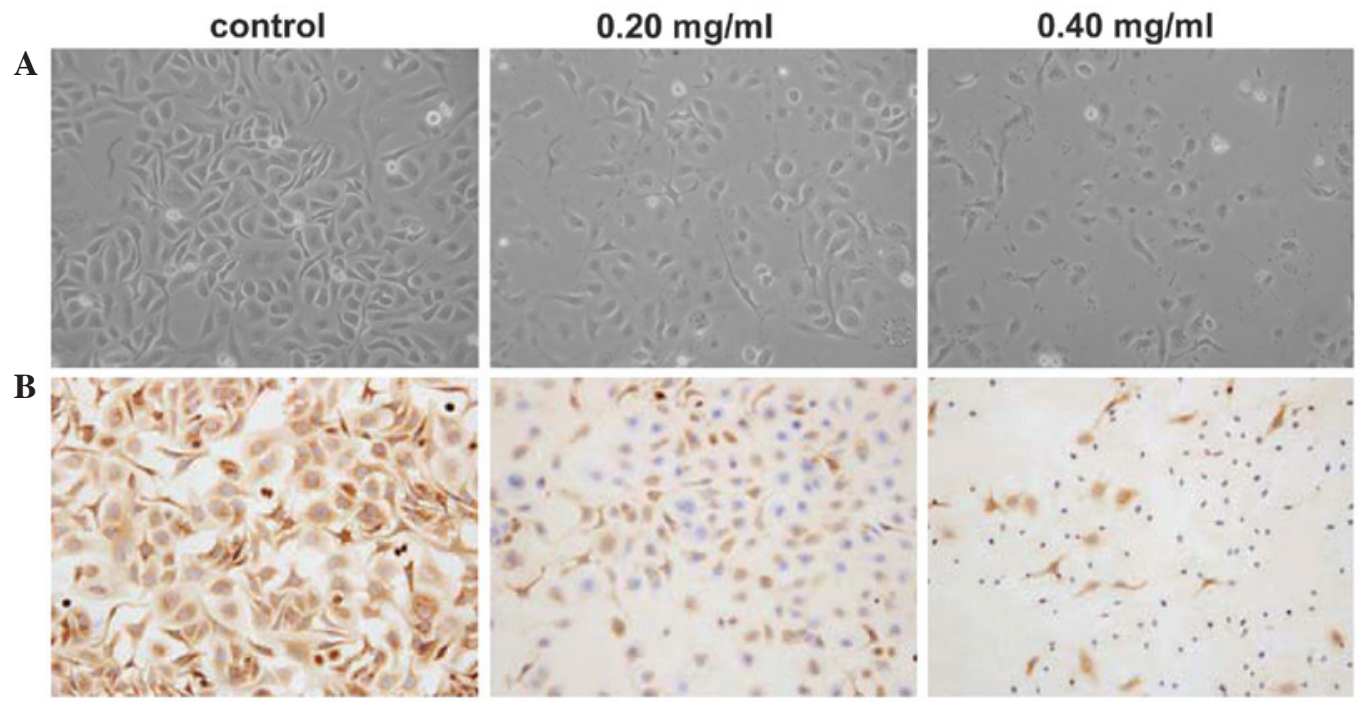

Figure 4. Morphological changes of SKOV3 ovarian cancer cells in response to 20(S)-protopanaxadiol saponins treatment. (A) Cells were treated with 0 (control), 0.2 or $0.4 \mathrm{mg} / \mathrm{ml}$ of PDS for $24 \mathrm{~h}$. The cells were then sampled and examined using an inverted microscope (magnification, $\mathrm{x} 200$ ). (B) The expression of OPN protein in SKOV3 cells. Expression of OPN protein appears predominantly cytoplasmic, with yellow or brown yellow staining (3, 3'-diaminobenzidine stain; hematoxylin counterstain; magnification, x200). OPN, osteopontin.

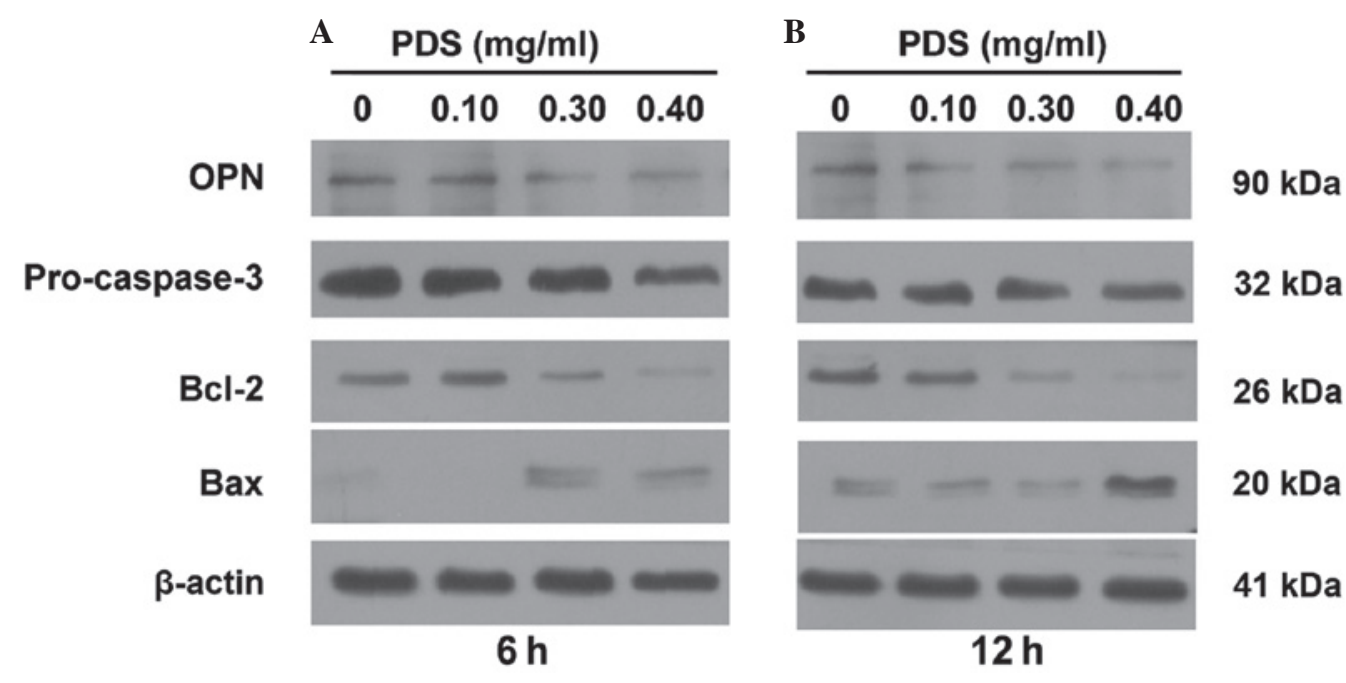

Figure 5. PDS modulates the of protein levels of OPN, pro-caspase-3, Bcl-2 and Bax in SKOV3 cells. (A) Cells were treated with the indicated concentrations of PDS for $6 \mathrm{~h}$. (B) The cells were treated with the indicated concentrations of PDS for $12 \mathrm{~h}$. $\beta$-actin was used normalize total proteins. PDS, 20(S)-protopanaxadiol saponins; OPN, osteopontin; Bcl-2, B-cell lymphoma 2; Bax, Bcl-2-like protein 4.

\section{Discussion}

The present study demonstrates that the migration of SKOV3 ovarian cancer cells was inhibited by dammarane glycoside 20(S)-protopanaxadiol, extracted from Panax notoginseng, and this was accompanied by a reduction in the expression of OPN protein in these cells.

Cell migration and invasion are fundamental aspects of cancer cell growth (13), requiring that neighbouring tumor cells loosen their intercellular junctions and that the ECM is proteolytically degraded (14). Cell migration and invasion are particularly important processes in ovarian cancer progression and have gained widespread attention (15). One such marker of tumor invasion and metastasis, OPN, may inhibit malignant tumor invasion and metastasis when its expression is suppressed (16). It has been reported previously that OPN downregulation significantly inhibits OPN protein expression in MDA-MB-231 breast cancer cells, reduces cell proliferation, and increases cell sensitivity to doxorubicin (17). The present study reveals that PDS decreased OPN protein in a dose-dependent manner. It is reasonable to postulate that the downregulation of OPN protein by PDS may also decrease cell-matrix adhesion. Therefore, if the factors that bind to these regulatory elements were blocked, the migration of SKOV3 cells could possibly be inhibited. Furthermore, PDS may not only be a potential agent for ovarian cancer treatment, but may also be applicable in preventing primary or metastatic tumors. Furthermore, pro-caspase-3 and Bcl-2 were observed to be downregulated by PDS and Bax was upregulated in the present study, which was also reported by Park et al (5).

In summary, the metastasis of ovarian cancer was significantly inhibited by PDS, partially due to the inhibition of 
tumor-induced cell invasion and migration. This may be associated with a decrease of OPN expression in the ovarian cancer cells.

\section{Acknowledgements}

The present study was financially supported by Yunnan Educational Committee Grant (grant no. 09ZZ113) and Key Project of the Applied Basic Research Programs of the Science and Technology Department of Yunnan Province (grant no. 2013FC006). The authors would like to thank Mr. Lloyd Zhao (School of Medicine, Duke University, Durham, NC, USA) for revising the English language of the manuscript.

\section{References}

1. Banerjee $\mathrm{S}$ and Gore $\mathrm{M}$ : The future of targeted therapies in ovarian cancer. Oncologist 14: 706-716, 2009.

2. Jiang B, Wang C, Han Y, Hu X, Zheng L and Zhao Y: Isolation and identification of minor bioactive saponins from the leaves of Panax notoginseng. Zhong Yao Cai 27: 489-491, 2004 (In Chinese).

3. Sun H, Yang Z and Ye Y: Structure and biological activity of protopanaxatriol-type saponins from the roots of Panax notoginseng. Int Immunopharmacol 6: 14-25, 2006.

4. Popovich DG and Kitts DD: Structure-function relationship exists for ginsenosides in reducing cell proliferation and inducing apoptosis in the human leukemia (THP-1) cell line. Arch Biochem Biophys 406: 1-8, 2002.

5. Park SC, Yoo HS, Park C, Cho CK, Kim GY, Kim WJ, Lee YW and Choi YH: Induction of apoptosis in human lung carcinoma cells by the water extract of Panax notoginseng is associated with the activation of caspase-3 through downregulation of Akt. Int J Oncol 35: 121-127, 2009.

6. Popovich DG and Kitts DD: Ginsenosides 20(S)-protopanaxadiol and $\mathrm{Rh} 2$ reduce cell proliferation and increase sub-G1 cells in two cultured intestinal cell lines, Int-407 and Caco-2. Can J Physiol Pharmacol 82: 183-190, 2004.
7. Chen G, Yang M, Nong S, Yang X, Ling Y, Wang D, Wang X and Zhang W: Microbial transformation of 20(S)-protopanaxadiol by Absidia corymbifera. Cytotoxic activity of the metabolites against human prostate cancer cells. Fitoterapia 84: 6-10, 2013.

8. Rittling SR and Chambers AF: Role of osteopontin in tumour progression. Br J Cancer 90: 1877-1881, 2004.

9. Tilli TM, Franco VF, Robbs BK, Wanderley JL, da Silva FR, de Mello KD, Viola JP, Weber GF and Gimba ER: Osteopontin-c splicing isoform contributes to ovarian cancer progression. Mol Cancer Res 9: 280-293, 2011.

10. Wei S and Lu XY: Effects of RNA interference targeting OPN on the in vitro proliferation of human ovarian cancer cell line HO-8910PM. Acta Acad Med Sinicae XuZhou 30: 504-510, 2010 (In Chinese).

11. Zhang X, Samadi AK, Roby KF, Timmermann B and Cohen MS: Inhibition of cell growth and induction of apoptosis in ovarian carcinoma cell lines $\mathrm{CaOV} 3$ and SKOV3 by natural withanolide Withaferin A. Gynecol Oncol 124: 606-612, 2012.

12. Xiao X, Zou J, Bui-Nguyen TM, Bai P, Gao L, Liu J, Liu S, Xiao J, Chen X, Zhang X and Wang H: Paris saponin II of Rhizoma raridis - a novel inducer of apoptosis in human ovarian cancer cells. Biosci Trends 6: 201-211, 2012.

13. Liotta LA, Tryggvason K, Garbisa S, Hart I, Foltz CM and Shafie S: Metastatic potential correlates with enzymatic degradation of basement membrane collagen. Nature 284: 67-68, 1980.

14. Comoglio PM and Trusolino L: Invasive growth: From development to metastasis. J Clin Invest 109: 857-862, 2002.

15. Tang L and Li Y: Inhibitory effects of natural food dyes genistein on invasion of SKOV3 human ovarian carcinoma cells in vivo and in vitro. Adv Mat Res 781-784: 1152-1155, 2013.

16. Kim JH, Skates SJ, Uede T, Wong KK, Schorge JO, Feltmate CM, Berkowitz RS, Cramer DW and Mok SC: Osteopontin as a potential diagnostic biomarker for ovarian cancer. JAMA 287: 1671-1679, 2002.

17. Yang L, Wei L, Zhao W, Wang X, Zheng G, Zheng M, Song X and Zuo W: Down-regulation of osteopontin expression by RNA interference affects cell proliferation and chemotherapy sensitivity of breast cancer MDA-MB-231 cells. Mol Med Rep 5: 373-376, 2012 